\section{Polynomial perception of exponential growth: A reply to Jones}

\author{
W. A. WAGENAAR \\ Institute for Perception \\ Soesterberg, The Netherlands
}

The note by Gregory Jones (Jones, 1977) is very interesting, since it contains some nice demonstrations of misperception. First, the polynomials produced by Jones predict responses about four times as high as the actually observed medians, using as many parameters as we needed for three dataplots. Second, Jones fails to explain the vast individual differences, which we were able to attribute to one single parameter. Third, Jones limited his attack to the results of Experiment I, Group 1, b series. Polynomial extrapolation can hardly account for the results of Experiment I, Group 2, or the c series of all groups, or for the results of Experiments II (all groups), III, and IV.

The effects not accounted for by Jones' model are the following: (a) The responses of Group 2 (yearby-year extrapolation) follow exponential curves quite nicely, but with an exponent which is markedly below the exponent in the starting series. Polynomial extrapolation can never produce the sharp inflection points that are demonstrated in our Figure 3. (b) When a constant is added to the starting series $(103,107,120,155,248$ instead of $3,7, \ldots 148)$, predictions drop dramatically (our Figure 2). Polynomial extrapolation would result in an increase of the predictions. (c) The results of Experiment II (graphical stimuli) are in no way near the predictions of Jones; even the third quartiles are a factor of 10 off, which is quite something if someone predicts population growth or the cost of living. (d) The results of Experiments III and IV (prior instructions and experience) show some marked effects on the willingness to produce large numbers but not on sensitivity to growth. Jones' claim that subjects performed optimally does not allow for such an effect.

Generally, it must be said that a description of the strategies in terms of fitting fourth-grade polynomials is quite unattractive, as it requires subjects to identify quartic components in a five-number series, which is psychologically implausible. Compare these number series:

$\begin{array}{llllll}\text { (a) } & 1 & 10 & 38 & 85 & 151 \\ \text { (b) } & 5 & 6 & 17 & 58 & 149 \\ \text { (c) } & 3 & 7 & 20 & 55 & 148\end{array}$

Would an average subject detect that the series were, respectively, quadratic, cubic, and quartic? Even a statistical test could not distinguish between these functions. I am afraid Jones was tempted to suggest fourth-grade polynomials because of the five-number series. Actually, the quadratic and cubic functions also provide very nice fits. Would Jones suggest taking ninth-grade polynomials if 10 starting numbers were used?

In conclusion, I think Jones' reinterpretation is quite insufficient as it accounts for only a minority of the results.

\section{REFERENCES}

JONES, G. V. Polynomial perception of exponential growth. Perception \& Psychophysics, 1977, 21, 197-198.

WagenaAr, W. A., \& SAgaria, S. D. Misperception of exponential growth. Perception \& Psychophysics, 1975, 18, 416-422.

(Received for publication December $31,1976$. 